\title{
Breaking the delay-bandwidth limit in a photonic structure
}

\author{
QIANFAN XU, PO DONG AND MICHAL LIPSON* \\ School of Electrical and Computer Engineering, Cornell University, 411 Phillips Hall, Ithaca, New York 14853, USA \\ *e-mail: lipson@ece.cornell.edu
}

Storing light on-chip, which requires that the speed of light be significantly slowed down, is crucial for enabling photonic circuits on-chip. Ultraslow propagation ${ }^{1-3}$ and even stopping ${ }^{4,5}$ of light have been demonstrated using the electromagnetically induced transparency effect in atomic systems ${ }^{1,3-5}$ and the coherent population oscillation effect in solid-state systems ${ }^{2}$. The wavelengths and bandwidths of light in such devices are tightly constrained by the property of the material absorption lines, which limits their application in information technologies. Various slow-light devices based on photonic structures have also been demonstrated ${ }^{6-10}$; however, these devices suffer a fundamental trade-off between the transmission bandwidth and the optical delay. It has been shown theoretically ${ }^{11-13}$ that stopping light on-chip and thereby breaking the fundamental link between the delay and the bandwidth can be achieved by ultrafast tuning of photonic structures. Using this mechanism, here we report the first demonstration of storing light using photonic structures on-chip, with storage times longer than the bandwidth-determined photon lifetime of the static device. The release time of the pulse is externally controlled.

The optical delay in a passive photonic structure is fundamentally limited by the bandwidth of the structure; to break this limit, dynamic tuning of the optical structure needs to be used. This can be understood by considering an optical resonator coupled to an adjacent waveguide. In such a structure, light coupled into the resonator from the waveguide will continuously couple back into the waveguide and travel forward. To hold the light in the resonator indefinitely, the resonator has to be completely decoupled from the waveguide. However, by definition, it is impossible to couple light into the completely decoupled resonator. Therefore, storing light using resonators requires the following steps: (1) coupling light from the waveguide into a resonator with a high optical coupling to the waveguide (we call this an open cavity); (2) storing light inside the resonator by dynamically tuning the coupling between the resonator and the waveguide to zero (we call it a closed cavity) while light is inside the resonator; and (3) releasing the stored light, by dynamically tuning the coupling between the resonator and the waveguide back to a high value so that the cavity is open again. The key to this operation is the ultrafast tuneability of the coupling between an optical cavity and an optical waveguide with a tuning time much shorter than the photon lifetime of the cavity.

Tuning of an integrated optical cavity in a timescale below nanoseconds is usually achieved through tuning the refractive index of the material forming the cavity. However, in traditional resonator structures, tuning the refractive index usually results in a wavelength shift of the resonance and only a minor change in the resonator's coupling to the adjacent waveguides. Here, to externally control the coupling of a resonator, we use the device shown in Fig. 1a, which holds a high-quality-factor $(Q)$ resonant mode owing to the coherent interference between two low- $Q$ ringresonator modes ${ }^{9,10,14-16}$. This high- $Q$ mode circulates in both ring resonators following the direction of the dashed arrows in Fig. 1a, and will be called the supermode hereafter. The coupling of this supermode and the adjacent waveguides is determined by the detuning between the two ring resonators ${ }^{9,10}$, which depends on the refractive index of the silicon forming each ring. The smaller the detuning, the lower the coupling. If the two ring resonances are identical, the supermode can be completely isolated from the waveguide, forming a closed cavity. We have recently showed fast tuning of the resonant wavelength of silicon resonators ${ }^{17-19}$ through the free-carrier dispersion effect ${ }^{20}$. Using the same mechanism, we can now dynamically tune the coupling of the supermode, which enables the storage of light on-chip.

The structure shown in Fig. la was fabricated on a silicon-oninsulator platform ${ }^{9}$. The radius of each ring resonator is $7 \mu \mathrm{m}$. The centre-to-centre distance between the two ring resonators is $22 \mu \mathrm{m}$. Therefore, the total length of the device is less than $40 \mu \mathrm{m}$.

Figure $1 \mathrm{~b}-\mathrm{g}$ shows the measured spectra and waveforms at the three stages of the storing and releasing operations. Figure $1 \mathrm{~b}$ shows the transmission spectrum of the structure in the initial state of the device. The detuning between the two ring resonances is $\lambda_{\mathrm{A}}-\lambda_{\mathrm{B}}=0.35 \mathrm{~nm}$, where $\lambda_{\mathrm{A}}$ and $\lambda_{\mathrm{B}}$ are the resonant wavelengths of rings $\mathrm{A}$ and $\mathrm{B}$ respectively. The full-width at half-maximum bandwidth of the supermode is $\sim 0.09 \mathrm{~nm}$, corresponding to $\sim 11 \mathrm{GHz}$. This state of the resonator, with relatively high input bandwidth, is used as the open-cavity state to couple light into the cavity. A 14 ps gaussian pulse, with the centre wavelength aligned with the supermode peak, is sent into the device. If no dynamic tuning of the cavity occurs, light leaks out of the cavity continuously as it couples into the cavity. Figure 1e shows the waveform of the output pulse where the tail of the output pulse follows an exponential delay curve (the dashed line) with a time constant of $18.6 \mathrm{ps}$, determined by the photon lifetime of this open cavity.

To close the cavity and store the pulse, the two ring resonators are tuned into resonance in a timescale shorter than the opencavity photon lifetime, by injecting a 1.5 ps optical control pulse at a wavelength of $415 \mathrm{~nm}$ on top of ring A. This control pulse is absorbed by the silicon ring, generates free carriers and blueshifts $\lambda_{\mathrm{A}}$ owing to the free-carrier dispersion effect in silicon ${ }^{18,20}$. The measured transmission spectrum of the closed cavity is shown as circles in Fig. 1c. The sharp peak from the supermode is absent in the spectrum. This is because the supermode is decoupled from the 


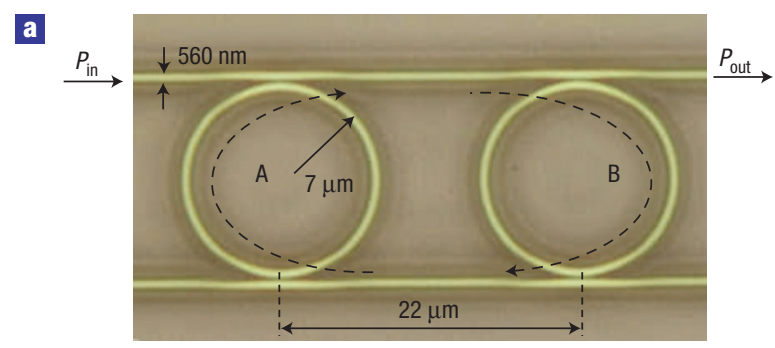

b

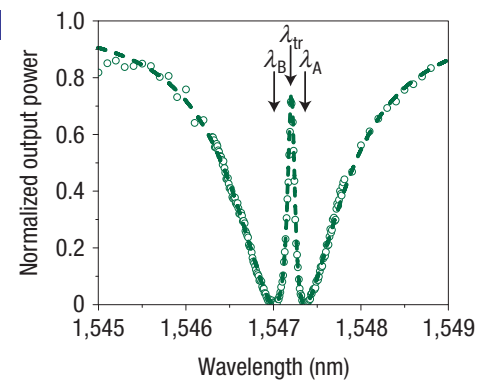

G

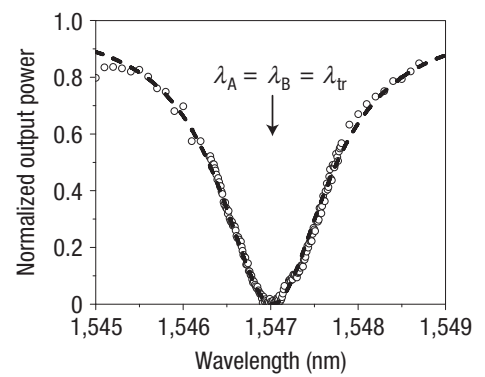

d

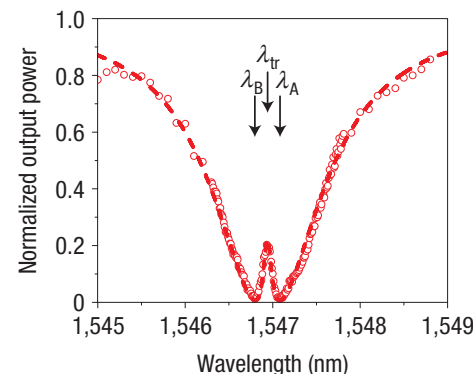

e

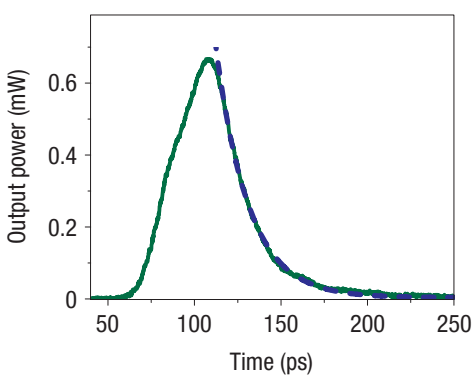

f

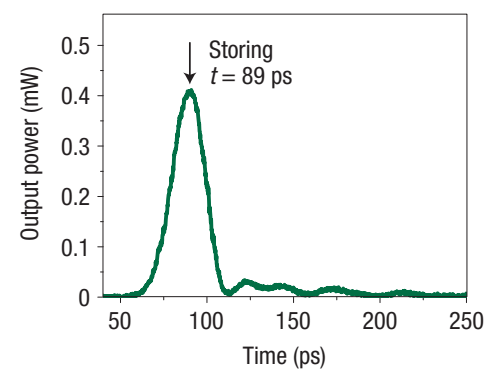

g

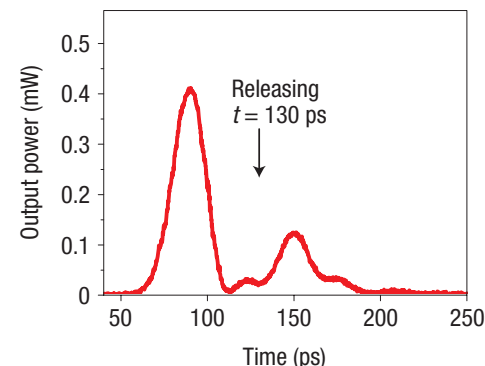

h

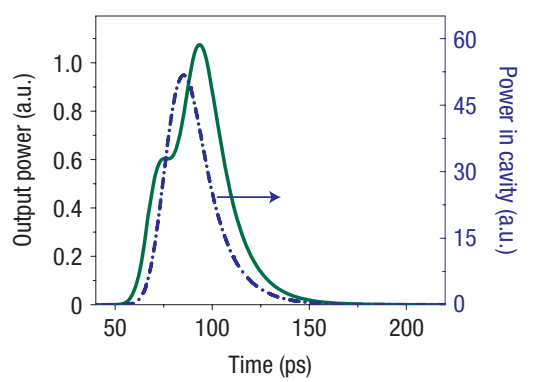

I

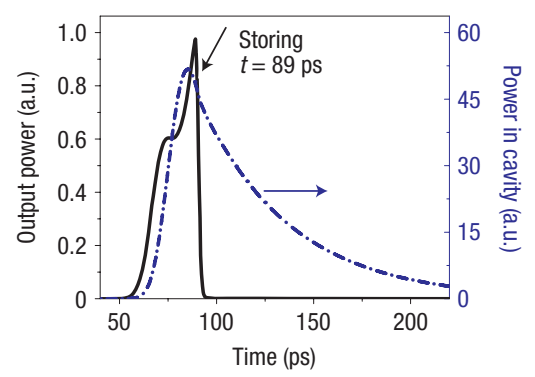

I

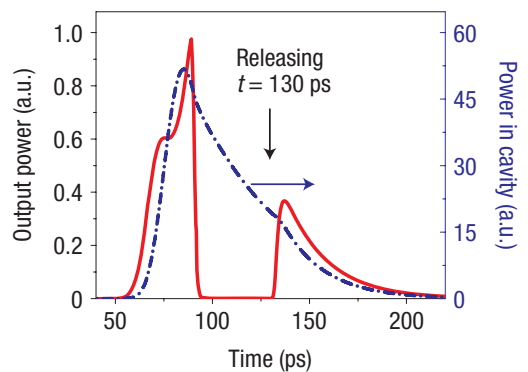

Figure 1 The device structure and the storing light operation. a, Top-view microscopic picture of the fabricated device. The device dimensions and the direction of the light travelling in the device are shown. $\mathbf{b}-\mathbf{d}$, Transmission spectra for the three stages of the storing and releasing operations: spectrum of the open cavity (b); spectrum of the closed cavity after the first control pulse (c); spectrum of the reopened cavity after the second control pulse (d). The circles are the measured data and the dashed lines show the spectra fitted to a theoretical model. $\mathbf{e}-\mathbf{g}$, Measured output waveforms for the three stages of the storing and releasing operations: output waveform without dynamic tuning (e), the dashed line is an exponential fit to the tail of the output pulse; output waveform with the first control pulse at 89 ps, which closes the cavity (f); output waveform with the first control pulse at $89 \mathrm{ps}$ and the second control pulse at $130 \mathrm{ps}(\mathbf{g})$. $\mathbf{h}-\mathbf{j}$, Simulated dynamics of the output optical power and the intracavity optical power: without dynamic tuning (h); with the control pulse at $89 \mathrm{ps}$ (i); with the first control pulse at $89 \mathrm{ps}$ and the second control pulse at $130 \mathrm{ps}(\mathbf{j})$.

waveguide, which implies that we cannot observe its existence from the transmission of the waveguide. The waveform of the output signal is shown in Fig. 1f, where the injection time of the control $(t=89 \mathrm{ps})$ is marked by an arrow. It can be seen that the output power drops to almost zero once the cavity is closed by the control pulse. This drop in transmission occurs because the optical power is now stored inside the cavity, instead of leaking out. The cavity can be kept in the closed state for hundreds of picoseconds before the generated carriers recombine and the ring resonance shifts back to its original state, because the carrier lifetime in these ring resonators is measured to be 700 ps. Refreshing control pulses with a time interval of less than 700 ps can be used to maintain the carrier density in ring $\mathrm{A}$, if a longer storage time is needed.

To release the stopped light following a given delay, we can reopen the cavity using another control pulse on top of ring B, and blue-shift its resonance. Following this second control pulse, the two ring resonances are again detuned from each other, and the supermode reappears on the transmission spectrum (Fig. 1d). 

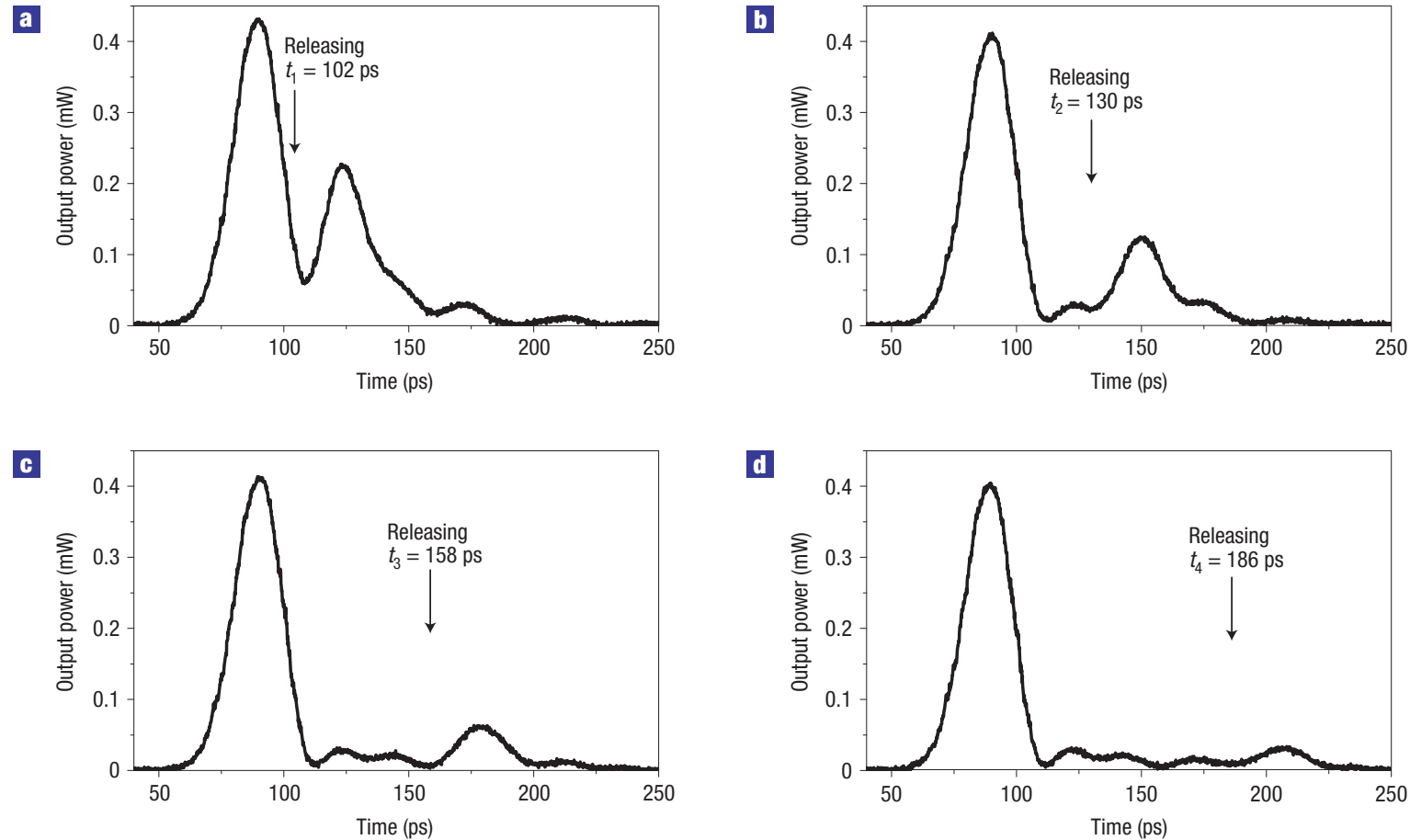

Figure 2 Output waveforms with different storage times. The first control pulse is fixed at $89 \mathrm{ps}$. The storage time is determined by the timing of the second control pulse for releasing the stored light. a, Releasing at 102 ps; storage time $=13$ ps. b. Releasing at 130 ps; storage time $=41$ ps. c, Releasing at 158 ps; storage time $=69 \mathrm{ps}$.

d, Releasing at $186 \mathrm{ps}$; storage time $=97 \mathrm{ps}$.

Figure $1 \mathrm{~g}$ shows the waveform of the output signal when the second control pulse is delayed by 41 ps from the first control pulse, where $41 \mathrm{ps}$ is the storage time. It can be seen that a released pulse appears after the second control pulse. The timing of the release of light is completely determined externally, by the timing of the second control pulse. Figure 2 shows the output waveforms when the delay between the second control pulse and the first control pulse (storage time) is varied over a span of 84 ps. It can be seen that the timing of the released pulses can indeed be tuned with the delay of the second control pulse. Note that the supermode peak in Fig. 1d has less transmission than the original supermode peak in Fig. $1 b$ because of the extra free-carrier absorption $\operatorname{loss}^{20}$ in the reopened cavity.

The spectra of the device in all three stages are fitted with a frequency-domain model $^{9}$, and are shown as dashed lines in Fig. 1b-d. The parameters of the device ${ }^{9}$, including the intrinsic $Q(=143,000)$ and coupling $Q$ of the ring resonators, the freecarrier absorption loss and the resonant wavelengths, $\lambda_{A}, \lambda_{B}$ and $\lambda_{\mathrm{tr}}$, at each stage are obtained from the fittings. These parameters are used in a time-domain dynamic model based on the transfermatrix method to simulate the optical power inside the resonators and the output optical power, when the tuning scheme is the same as that in the experiment. The results are shown in Fig. 1h-j. It can be seen that the measured waveforms agree well with the simulated ones, except that the measured waveform has a longer transition time and some ripples after sharp transitions due to the imperfect detector response, which is not present in the simulation. From Fig. 1h, it can be seen that the intracavity optical power and the output pulse decay at the same rate, indicating that the decay time of the output pulse shown in Fig. 1e is indeed equal to the photon lifetime of the open cavity. Figure 1i shows that, when the cavity is closed, the output power of the device immediately drops to zero, and the decay rate of the intracavity optical power reduces compared with that of the open cavity, because the cavity is decoupled from the waveguides.

For a completely closed cavity, light could be stored inside the cavity indefinitely if the cavity was lossless. In the experiment, the stored optical energy continuously drops owing to both scattering loss characterized by the intrinsic $Q$ of the cavity and extra loss from the free-carrier absorption effect. Note that these losses can be overcome by optimizing the fabrication process ${ }^{21}$ and the tuning process $^{22}$. The squares in Fig. 3 show the experimentally measured relationship between the peak power of the released pulse and the storage time. These squares can be fitted very well with an exponential decay curve (the solid line), which agrees well with the simulated intracavity power decay curve of the closed cavity shown in Fig. 1i. The time constant of the decay curve in Fig. 3, which is measured to be $43.3 \mathrm{ps}$, is the photon lifetime of the closed cavity. Despite the extra loss from the free-carrier absorption effect, the photon lifetime of the closed cavity is longer than that of the open cavity (18.6ps), enabling the storage time to overcome the traditional delay-bandwidth product limit. Note that part of the input light leaks into the output waveguide before the cavity is closed, which is independent of the releasing time and is shown as initial peaks in Fig. 2. This is due to the limited optical path length of the device, whereas theoretical studies show no such leakage in multiple cascaded cavities ${ }^{11}$.

The storing of light operation shown here is associated with a bandwidth compression of the light ${ }^{13}$. When the resonator is adiabatically tuned, the spectral property of the light that is circulating in the resonator during the tuning process follows the spectral property of the resonance mode ${ }^{23,24}$. In this experiment, when the cavity is closed, and the linewidth of the supermode is compressed, the linewidth of the light stored in the closed 


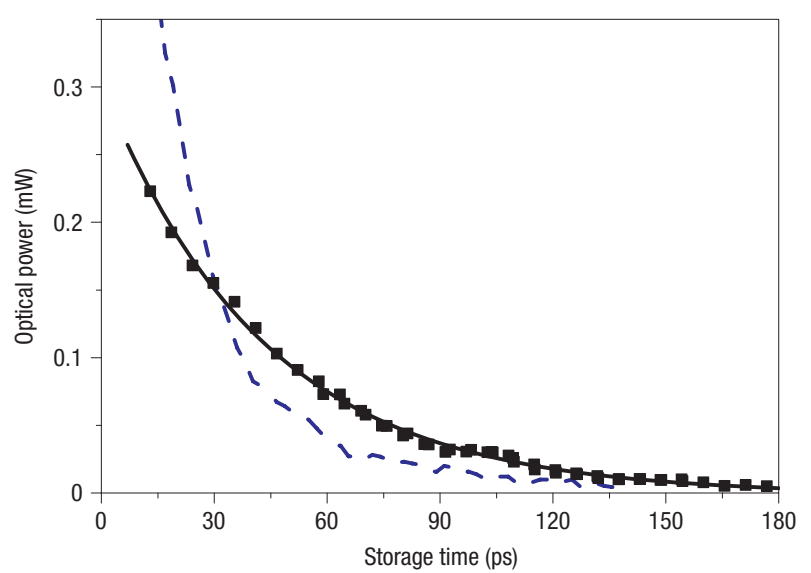

Figure 3 The temporal decay of optical energy stored in the cavity. The squares are the measured peak powers of the released pulses with different storage times. The solid line is an exponential decay curve with a time constant of $43.3 \mathrm{ps}$ fitted to the squares, which is proportional to the temporal decay of the optical energy in the closed cavity. The dashed line is the tail of the output waveform of the device without any control pulse, which is proportional to the temporal decay of the optical energy in the open cavity.

cavity is also compressed ${ }^{13}$. When the cavity is reopened, the supermode wavelength, $\lambda_{\mathrm{tr} 2}$, is blue-shifted from the original resonant wavelength, $\lambda_{\mathrm{trl}}$. Therefore, the released pulse from the reopened cavity centres at $\lambda_{\mathrm{tr} 2}$, blue-shifted from the input pulse wavelength. We can compare the spectrum of the released pulse and that of the input pulse by comparing the spectra following different release times. The spectra measured following a longer storage time will have a smaller signature of the stored pulse owing to the losses in the closed cavity. Figure 4 shows the spectra of output signals with storage times of $35 \mathrm{ps}$ (solid line) and $108 \mathrm{ps}$ (dashed line), measured with an optical spectrum analyser. We can identify on the solid line a peak at $\lambda_{\mathrm{tr} 2}$ associated with the released pulse, which is blue-shifted from the main peak associated with the leaked input pulse. Note that to tune and to compress the bandwidth of the light without scattering to other resonant modes of the rings, the tuning of the ring needs to be adiabatic, that is, the tuning time needs to be much longer than $1 / \Delta \omega$ (ref. 24), where $\Delta \omega$ is the difference in angular frequency between resonant modes. For the device measured here, the free-spectral range is $12.1 \mathrm{~nm}$, corresponding to $1 / \Delta \omega=100 \mathrm{fs}$. The tuning time in the experiment is $\sim 1.5 \mathrm{ps}$, much longer than $1 / \Delta \omega$.

When the cavity is closed and the bandwidth of the light is compressed, the cavity mode stores the energy and the phase of the input optical pulse, but not the shape of the pulse. The spectral and temporal shape of the released pulse is determined by the property of the reopened cavity. This is evident from Fig. $1 \mathrm{j}$, where the released pulse has an exponential tail determined by the photon lifetime of the reopened cavity. To store the shape of an optical input, or to store multiple pulses, we would need to cascade multiple pairs of ring resonators ${ }^{11-13}$.

Finally, with our recent demonstration of fast electro-optic tuning of the ring resonances with built-in $\mathrm{p}-\mathrm{i}-\mathrm{n}$ junctions ${ }^{19,22}$, electrically controlled storage of light could be achieved. When the cavity closure is carried out by carrier extraction, the storage time is limited only by the intrinsic $Q$ of the individual ring resonator, completely independent of the input bandwidth of the device. With the recent demonstration of $Q \sim 4.8 \times 10^{6}$ in a silicon ring resonator ${ }^{25}$, a storage time of several nanoseconds is possible, while the input bandwidth is kept over $10 \mathrm{GHz}$.

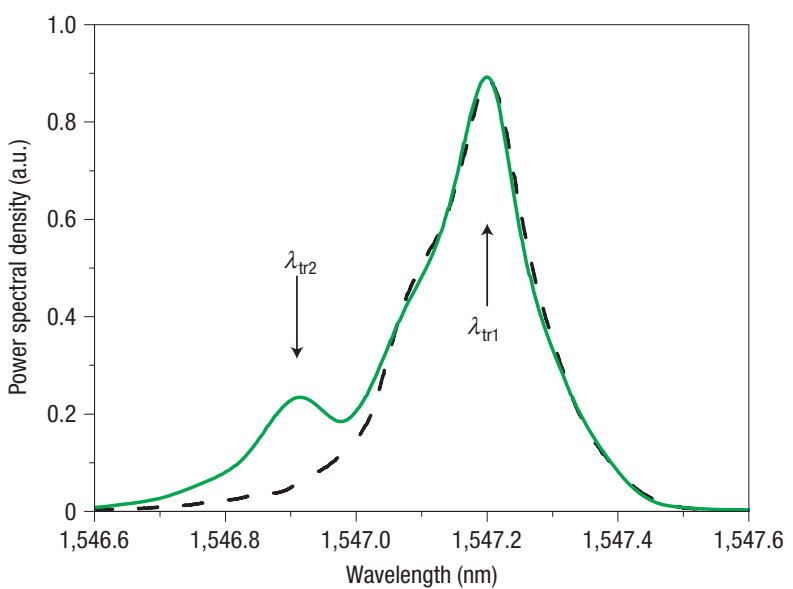

Figure 4 The spectra of the optical output. The spectra when the storage time is $35 \mathrm{ps}$ (green solid line) and $108 \mathrm{ps}$ (black dashed line).

\section{METHODS}

The resonances of ring resonators are tuned using control pulses injected out-of-plane. $1.5 \mathrm{ps}$ optical pulses at a wavelength of $830 \mathrm{~nm}$ with a repetition rate of $76 \mathrm{MHz}$ are generated by a Ti:sapphire mode-locked laser. These pulses are split using a beam splitter. One output is used to pump an optical parametrical oscillator to generate the probe pulses at $\sim 1,547 \mathrm{~nm}$. The other output is used to pump a beta-barium borate crystal for second-harmonic generation. The second-harmonic generation output at a wavelength of $415 \mathrm{~nm}$ is further split into two beams of control pulses, one is synchronized to the probe pulse to close the cavity, the other, with a tuneable delay, is used as the second control to reopen the cavity.

Both control beams are coupled into short optical fibres ( $\sim 1 \mathrm{~m}$ long). The other ends of the fibres are cleaved and aimed at the two rings from $\sim 45^{\circ}$ out-of-plane, and are brought very close to the device. Therefore, the spot size of each control beam is close to the size of the $10 \mu \mathrm{m}$ fibre core. Each control pulse is absorbed by silicon and generates free carriers in the ring and blue-shifts the resonant wavelength of the ring. The energy of each control pulse is $\sim 13 \mathrm{pJ}$. From the resonance shift, we estimate that $\sim 0.8 \mathrm{pJ}$ is absorbed by the silicon ring.

The output of the optical parametrical oscillator is coupled into a single-mode optical fibre and sent through a $0.25 \mathrm{~nm}$ grating filter, from which the 14 ps gaussian probe pulses are obtained. The probe pulses are set to the quasi-transverse-magnetic polarization and are sent into the silicon waveguide through a tapered lens fibre with a mode-field diameter of $3.8 \mu \mathrm{m}$. The output from the silicon waveguide is coupled back into the single-mode optical fibre using a lens and a fibre collimator. The silicon waveguide is $\mathrm{Z}$-shaped with a total length of $\sim 7 \mathrm{~mm}$. Both the straight waveguides and the curved waveguide forming the rings have a height of $250 \mathrm{~nm}$ and a width of $560 \mathrm{~nm}$. Nanotapers ${ }^{26}$ with a tip width of $80 \mathrm{~nm}$ are used in each side of the waveguide to reduce the coupling loss between the optical fibre and the waveguide. The fibre-to-fibre insertion loss of the device (at the wavelengths away from the ring resonances) is $\sim 7 \mathrm{~dB}$. Half of the output optical signal is sent to a fast detector with a $15 \mathrm{ps}$ rise and fall time and a sampling oscilloscope to record the time-domain traces shown in Figs 1e-g and 2. The other half of the output optical signal is sent to an optical spectrum analyser with a nominal resolution of $0.07 \mathrm{~nm}$ to record the spectra shown in Fig. 4.

To measure the transmission spectra of the device before and after each control pulse, a continuous-wave probe light generated by a tuneable laser is sent into the device. The time-domain traces of the output optical power are recorded by the fast detector and the sampling oscilloscope. Three time spans are selected out from the traces, which are the time before the first control pulse, the time 50-80 ps after the first control pulse and the time 50-100 ps after the second control pulse (the delay between the second and the first control pulse is $\sim 90 \mathrm{ps}$ in this measurement). For each wavelength, the average output power in these three time spans is used to plot the data points in the spectra in Fig. 1b-d, respectively. These time spans are chosen to minimize the artefacts 
from the detector response and the carrier recombination. However, as can be seen from Fig. 1f, the detector response shows small but long-lived ripples after a sharp transition. These ripples cause a slight distortion of the measured spectra in Fig. 1c,d at the original supermode peak wavelength $\left(\lambda_{\text {tr }}\right.$ in Fig. $\left.1 b\right)$.

\section{Received 15 November 2006; accepted 29 March 2007; published 22 April 2007.}

\section{References}

1. Hau, L. V., Harris, S. E., Dutton, Z. \& Behroozi, C. H. Light speed reduction to 17 metres per second in an ultracold atomic gas. Nature 397, 594-598 (1999).

2. Bigelow, M. S., Lepeshkin, N. N. \& Boyd, R. W. Superluminal and slow light propagation in a room-temperature solid. Science 301, 200-202 (2003).

3. Ghosh, S., Sharping, J. E., Ouzounov, D. G. \& Gaeta, A. L. Resonant optical interactions with molecules confined in photonic band-gap fibers. Phys. Rev. Lett. 94, 093902 (2005).

4. Liu, C., Dutton, Z., Behroozi, C. H. \& Hau, L. V. Observation of coherent optical information storage in an atomic medium using halted light pulses. Nature 409, 490-493 (2001).

5. Phillips, D. F., Fleischhauer, A., Mair, A., Walsworth, R. L. \& Lukin, M. D. Storage of light in atomic vapor. Phys. Rev. Lett. 86, 783-786 (2001).

6. Vlasov, Y. A., O'Boyle, M., Hamann, H. F. \& McNab, S. J. Active control of slow light on a chip with photonic crystal waveguides. Nature 438, 65-69 (2005).

7. Gersen, H. et al. Real-space observation of ultraslow light in photonic crystal waveguides. Phys. Rev. Lett. 94, 073903 (2005)

8. Poon, J. K., Zhu, L., DeRose, G. A. \& Yariv, A. Transmission and group delay of microring coupled-resonator optical waveguides. Opt. Lett. 31, 456-458 (2006).

9. $\mathrm{Xu}$, Q. et al. Experimental realization of an on-chip all-optical analogue to electromagnetically induced transparency. Phys. Rev. Lett. 96, 123901 (2006).

10. Xu, Q., Shakya, J. \& Lipson, M. Direct measurement of tunable optical delays on chip analogue to electromagnetically induced transparency. Opt. Express 14, 6463-6468 (2006).

11. Yanik, M. F., Suh, W., Wang, Z. \& Fan, S. Stopping light in a waveguide with an all-optical analogue of electromagnetically induced transparency. Phys. Rev. Lett. 93, 233903 (2004).

12. Yanik, M. F. \& Fan, S. Stopping light all-optically. Phys. Rev. Lett. 92, 083901 (2004).

13. Yanik, M. F. \& Fan, S. Stopping and storing light coherently. Phys. Rev. A 71, 013803 (2005).
14. Chu, S. T., Little, B. E., Pan, W., Kaneko, T. \& Kokebun, Y. Second-order filter response from parallel coupled glass microring resonators. IEEE Photon. Technol. Lett. 11, 1426-1428 (1999).

15. Matsko, A. B., Savchenkov, A. A., Strekalov, D., Ilchenko, V. S. \& Maleki, L. Interference effects in lossy resonator chains. J. Mod. Opt. 51, 2515-2522 (2004).

16. Emelett, S. \& Soref, R. Analysis of dual-microring-resonator cross-connect switches and modulators. Opt. Express 13, 7840-7853 (2005).

17. Almeida, V. R., Barrios, C. A., Panepucci, R. R. \& Lipson, M. All-optical control of light on a silicon chip. Nature 431, 1081-1084 (2004)

18. Almeida, V. R. et al. All-optical switching on a silicon chip. Opt. Lett. 29, 2867-2869 (2004).

19. Xu, Q., Schmidt, B., Pradhan, S. \& Lipson, M. Micrometre-scale silicon electro-optic modulator. Nature 435, 325-327 (2005).

20. Soref, R. A. \& Bennett, B. R. Electrooptical effects in silicon. IEEE J. Quantum Electron. 23 123-129 (1987).

21. Borselli, M., Johnson, T. J. \& Painter, O. Measuring the role of surface chemistry in silicon microphotonics. App. Phys. Lett. 88, 131114 (2006).

22. Preble, S. F., Xu, Q., Schmidt, B. S. \& Lipson, M. Ultrafast all-optical modulation on a silicon chip. Opt. Lett. 30, 2891-2893 (2005).

23. Notomi, M. \& Mitsugi, S. Wavelength conversion via dynamic refractive index tuning of a cavity. Phys. Rev. A 73, 051803 (2006).

24. Gaburro, Z. et al. Photon energy lifter. Opt. Express 14, 7270-7278 (2006).

25. Borselli, M. High-Q Microresonators as Lasing Elements for Silicon Photonics. Thesis, California Institute of Technology, Pasadena (2006).

26. Almeida, V. R., Panepucci, R. R. \& Lipson, M. Nanotaper for compact mode conversion. Opt. Lett. 28 1302-1304 (2003).

\section{Acknowledgements}

The authors thank S. Fan of Stanford University for fruitful discussions. This work was carried out in part at the Cornell Nano-Scale Science \& Technology Facility (CNF) and the Cornell Center for Nanoscale Systems (CNS).

Correspondence and requests for materials should be addressed to M.L.

Competing financial interests

The authors declare no competing financial interests.

Reprints and permission information is available online at http://npg.nature.com/reprintsandpermissions/ 